\title{
Postsorafenib systemic treatments for hepatocellular carcinoma: questions and opportunities after the regorafenib trial
}

\section{Future ONCOLOGY}

Francesco Tovoli ${ }^{* 1}$, Stefania De Lorenzo ${ }^{2}$, Maria Aurelia Barbera², Ingrid Garajova², Giorgio Frega ${ }^{2}$, Andrea Palloni ${ }^{2}$, Maria Abbondanza Pantaleo ${ }^{2,3}$, Guido Biasco ${ }^{2,3}$ \& Giovanni Brandi ${ }^{3}$

The search for systemic therapies for hepatocellular carcinoma has been characterized by difficulties and failures. Despite recent progresses, many issues are still to be settled. In particular, the development of drugs inhibiting different neoplastic pathways remains a priority for patients intolerant or resistant to antiangiogenic drugs. This task may be daunting, as previous failures extensively demonstrated. We aimed to identify the future perspective of postsorafenib trials analyzing the strengths and the critical points of past and currently undergoing studies, in the light of the most recent evidences in the field. We identified various points (including stratification, biomarkers, end points, radiologic criteria of response, treatment beyond radiologic progression) that should be considered by future trials to reduce the risks of failure.

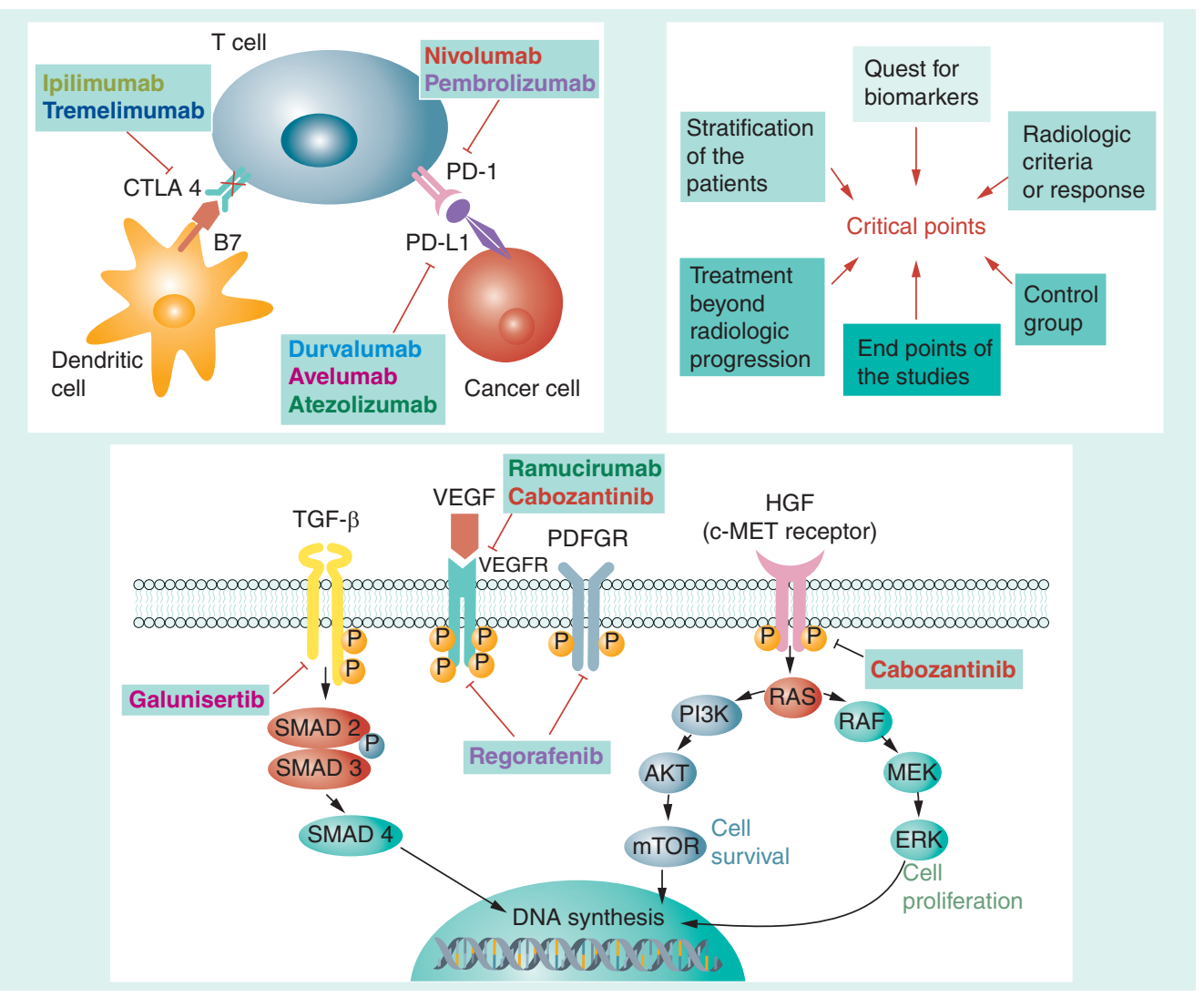

'Unit of Internal Medicine, Department of Medical \& Surgical Sciences, University of Bologna, Italy

${ }^{2}$ Unit of Oncology, Department of Experimental, Diagnostic \& Specialty Medicine, University of Bologna, Italy

${ }^{3}$ G. Prodi' Interdepartmental Center for Cancer Research (C.I.R.C.), University of Bologna, Italy

*Author for correspondence: francesco.tovoli2@unibo.it

Future Medicine part of 


\section{KEYWORDS}

- cabozantinib

- hepatocellular carcinoma

- immunotherapy

- nivolumab • ramucirumab

- RECIST • regorafenib

- sorafenib

First draft submitted: 7 April 2017; Accepted for publication: 19 May 2017; Published online: 11 July 2017

Hepatocellular carcinoma (HCC) is a significant clinical problem in the field of hepatology and oncology. Its incidence is on the rise in many western countries and a further increase of cases is expected in Central Europe and in the USA in the next years [1]. Unfortunately, the overall prognosis of HCC is still poor, resulting in 250,000-1,000,000 deaths per year [1]. Patients with advanced HCC had no effective therapies until 2008, when the SHARP trial demonstrated a clear benefit of sorafenib, a VEGFR inhibitor, compared with placebo in terms of overall survival (OS) and time to progression (TTP) [2]. Since then, sorafenib has become the recommended systemic therapy for HCC [3-6]. However, most patients treated with sorafenib experience a progression of their disease and/or side effects causing its permanent discontinuation [7].

Therefore, the identification of an effective second-line systemic treatment has been increasingly regarded a goal of paramount importance. After the success of the SHARP trial, a number of second-line randomized clinical trials (RCTs) tried to identify other effective anti-VEGFR agents. Examples of these failed trials include the BRISK (brivanib) [8] and the REACH studies (ramucirumab) [9] (Table 1).

These failures encouraged the development of molecules inhibiting antineoplastic pathways different from VEGFR. These efforts have still to be crowned with success. The EVOLVE-1 trial (everolimus) failed to demonstrate a survival benefit in patients treated with an inhibitor of mammalian target of rapamycin [10]. More recently codrituzumab, a monoclonal antibody against a liver cancer protein called glypican-3, also failed to improve the OS of HCC patients unresponsive or intolerant to sorafenib [11]. Additionally, the use of a novel anticancer enzyme (ADI-PEG20) that produces intratumor depletion of arginine equally failed to prolong life expectancy [12].

Most recently, tivantinib, a promising nonATP-competitive small molecule inhibitor of the tyrosine kinase c-MET, also failed its primary end point of improving $O S$ in patients with tumors exhibiting high MET-expression by immunochemistry [13]. Further, it also failed its end point of improving progression-free survival in a second Phase III pivotal trial performed in a Japanese MET-high population [14].

Fortunately, these failures did not discourage researchers. Most of the currently undergoing trials still aim to evaluate drugs which do not selectively block the VEGFR pathway. Cancer immunotherapy, for instance, has gained a vast popularity between hepatologists and oncologists.

Just as the attention of the researchers seemed to have moved far away from selective antiangiogenic agents, the perspectives of both patients and scientists were finally galvanized by the demonstration of efficacy of regorafenib, another anti-VEGFR drug.

The randomized, double-blind, parallelgroup, Phase III trial RESORCE trial enrolled 573 patients at 152 sites across 21 countries. Only progressors to sorafenib could be enrolled. On the contrary, patients who had not tolerated sorafenib at the minimum dose of $400 \mathrm{mg} / \mathrm{day}$ for at least 20 of the last 28 days of treatment were not enrolled due to the high risk of crossed intolerance. Enrolled patients were randomized $2: 1$ to receive either regorafenib $160 \mathrm{mg} /$ day

Table 1. Previously failed Phase III randomized clinical trials investigating second-line drugs for patients with unresectable hepatocellular carcinoma not amenable for locoregional treatments who progressed or were intolerant to sorafenib.

\begin{tabular}{|lll|}
\hline Name of the trial & Study drug & Primary end point \\
BRISK-PS & Brivanib vs placebo & No difference in OS (HR 0.89, 95\% Cl: 0.69-1.15, p=0.33) \\
\hline REACH & Ramucirumab vs placebo & No difference in OS (HR 0.87, 95\% Cl: 0.72-1.05, p=0.14) \\
\hline EVOLVE-1 & Everolimus vs placebo & No difference in OS (HR 1.05, 95\% Cl: 0.86-1.27, p=0.68) \\
\hline POLARIS2009-001 & ADI-PEG20 vs placebo & No difference in OS (HR 1.02, 95\% Cl: 0.85-1.23, p =0.88) \\
METIV-HCC & Tivantinib vs placebo & No difference in OS (further data still not released) \\
\hline JET-HCC & Tivantinib vs placebo & No difference in PFS (further data still not released) \\
\hline HCC: Hepatocellular carcinoma; OS: Overall survival; PFS: Progression-free survival.
\end{tabular}


or placebo. Randomization was stratified by geographical region, macrovascular invasion, extrahepatic spread, alfa-fetoprotein (AFP) concentration $(<400 \mathrm{ng} / \mathrm{ml}$ vs $=400 \mathrm{ng} / \mathrm{ml})$, and Eastern Cooperative Oncology Group performance status (0 vs 1 ). Patients in the regorafenib treatment arm showed a significant survival gain compared with placebo (median OS 10.6 vs 7.8 months) with a reduction of the risk of death of 37\% (hazard ratio 0.63 [CI: 0.50-0.79], p < 0.001 ) [9]. Of note, drug-related adverse events led to interruptions or dose reductions in 54\% of regorafenib-treated patients [15].

The role of antiangiogenic drugs was further affirmed by a very recent press release regarding a large Phase III frontline HCC trial, in which the multikinase inhibitor lenvatinib resulted noninferior to sorafenib in terms of OS [16].

Still, the demand for new drugs is still high, especially for patients intolerant to sorafenib and/or regorafenib. Additionally, a number of studies suggested that a carefully selected subset of the Child-Pugh B population may benefit from sorafenib (i.e., Child-Pugh B7) [17,18]. Consequently, the search for second-line systemic treatments able to prolong life expectancy of Child-Pugh B7 patients has a scientific rationale and represents an open problem.

The aim of this review is to identify the future lines of research in the field of postsorafenib systemic treatments for HCC: analyzing the ongoing second line trials for advanced HCC; and assessing the pitfalls and the open problems in the design of current and future trials.

\section{Materials \& methods}

We adopted a search strategy on PubMed based on search for the following string 'CARCINOMA, HEPATOCELLULAR' [MESH] AND 'SORAFENIB' [SUPPLEMENTARY CONCEPT] AND SURVIVAL published in English between 1 January 2008 and 31 March 2017, finding 634 entries. Then, we searched Clinicaltrials.gov with the terms 'HEPATOCELLULAR CARCINOMA' and 'LIVER CANCER' to identify ongoing Phase II and Phase III postsorafenib clinical trials, finding 201 entries. We then made a selection of papers of interest based on title and - optionally - on abstract and added studies known to the authors or cited in previous reviews. We finally selected a total number of 65 studies, divided in two distinct chapters: ongoing clinical trials, and current changes in the design of clinical trials.

\section{Results \\ - Ongoing clinical trials}

Ramucirumab (anti-VEGFR)

The interest toward antiangiogenetic agents is still high, especially after the success of the SHARP and RESORCE trial. Ramucirumab is a recombinant IgG1 monoclonal antibody and a VEGFR-2 antagonist [9] (Figure 1). Its efficacy and safety as a second-line treatment for unresectable HCC was investigated in the aforementioned REACH study. The trial failed to demonstrate a significant benefit in OS in the ramucirumab treatment arm. However, in a subgroup analysis of patients with a baseline AFP concentration $\geq 400 \mathrm{ng} / \mathrm{ml}$ ramucirumab showed a meaningful effect on OS (HR 0.674; median OS 7.8 vs 4.2 months; $\mathrm{p}=0.006$ ) [9]. The survival analysis of patients with baseline high AFP was predefined in the statistical analysis plan.

Unfortunately, the original study design did not stratify patients according to AFP values at the time of inclusion. Thus, the currently ongoing Phase III REACH-2 study (NCT02435433) compares ramucirumab versus placebo in patients with AFP > $400 \mathrm{ng} / \mathrm{ml}[19]$.

\section{Cabozantinib (combined VEGFR \& MET-}

inhibitor)

The rationale for a dual VEGFR/MET blockade is supported by the consolidated evidence that resistance to VEGFR-targeted therapies may arise from the upregulation of alternative proangiogenic and proinvasive signaling pathways, including the MET pathway. As such, combined inhibition of the VEGFR and MET resulted in an increased efficacy compared with that achieved inhibiting either pathway alone in some tumor models [20-23].

Cabozantinib (XL-184) is an orally bioavailable tyrosine kinase inhibitor with strong activity against both MET and VEGFR2 (Figure 1). It also inhibits many other receptor tyrosine kinases (such as RET, KIT, AXL and FLT3) that have been implicated in neoplastic pathobiology [24]. In 2012 cabozantinib was approved by the US FDA for the treatment of progressive metastatic medullary thyroid cancer, following a successful Phase III trial [25].

A possible therapeutic role of cabozantinib in HCC has been investigated in a Phase II randomized discontinuation study. The majority of subjects $(80 \%)$ had received a prior systemic therapy and about half $(51 \%)$ had received prior sorafenib. After 12 weeks, the disease control 


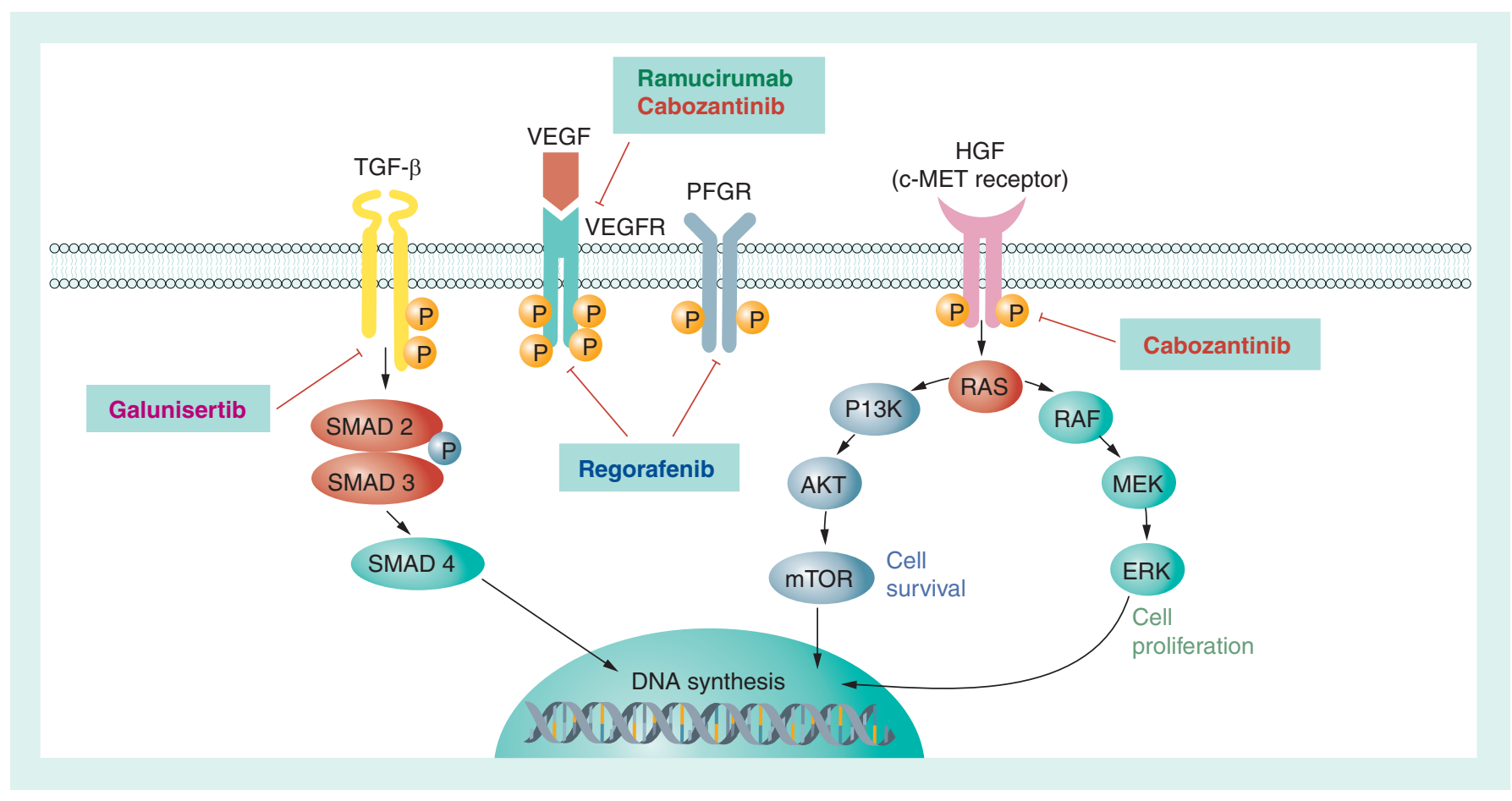

Figure 1. Principal tumor pathways inhibited by systemic drugs for hepatocellular carcinoma.

rate was $66 \%$. The median OS from the initial cabozantinib dose was 11.5 months (95\% CI: 7.3-15.6) in the whole study population and 12.9 months (95\% CI: 10-16.8) among sorafenibpretreated subjects [26]. These results warranted the currently ongoing Phase III, Randomized, Double-blind, Controlled Study of Cabozantinib versus Placebo in Subjects with Hepatocellular Carcinoma Who Have Received Prior Sorafenib (CELESTIAL; NCT01908426) [27].

The most interesting feature of this study is the possibility to enroll also patients who experienced two previous lines of therapy, making it a second-/third-line clinical trial [27]. While this feature may add some problems in the interpretation of the study data, cabozantinib could become the third-line systemic treatment for advanced HCC in the eventuality of a successful trial. The recruitment is still open and the final results are not expected before 2018 .

Immune checkpoint inhibitors

The availability of clinical trials investigating immunomodulating agents was without doubt the main innovation in the field of the systemic treatments for HCC in the last year. The best known molecules in immunotherapy are nivolumab, a inhibitor of the lymphocytes programmed death 1 (PD1) receptor, and ipilimumab, a cytotoxic T-lymphocyte antigen 4 (CTLA-4). More recently, a different PD1 inhibitor (pembrolizumab) and the antagonists of PD1 ligand 1 (PD-L1) durvalumab, avelumab and atezolizumab have been developed [28]. All of these drugs inhibit a immune checkpoint exploited by the tumor cells to protect themselves from the immune system recognition and destruction [28,29] (Figure 2).

Enthusiasms are mainly due to previous clinical studies that demonstrated the efficacy and the safety of nivolumab, alone or in association with ipilimumab, in the treatment of metastatic melanoma [30] and non-small-cell lung carcinoma $[31,32]$. Consequently, the announcement of a multicohort Phase I-II trial of nivolumab alone or combined with ipilimumab in HCC (Checkmate-040, NCT01658878) was greeted with genuine satisfaction. Basically, the CA209-040 trial includes: dose escalation/ expansion cohorts; a cohort for a randomized 1:1 comparison versus sorafenib as a frontline therapy; a combination therapy cohort with ipilimumab for patients progressing or intolerant to sorafenib; and a cohort reserved to patients with Child-Pugh B HCC [33,34].

Expectations were met by results from the first two cohorts, evidencing a unprecedented overall response rate (ORR) for a systemic therapy 


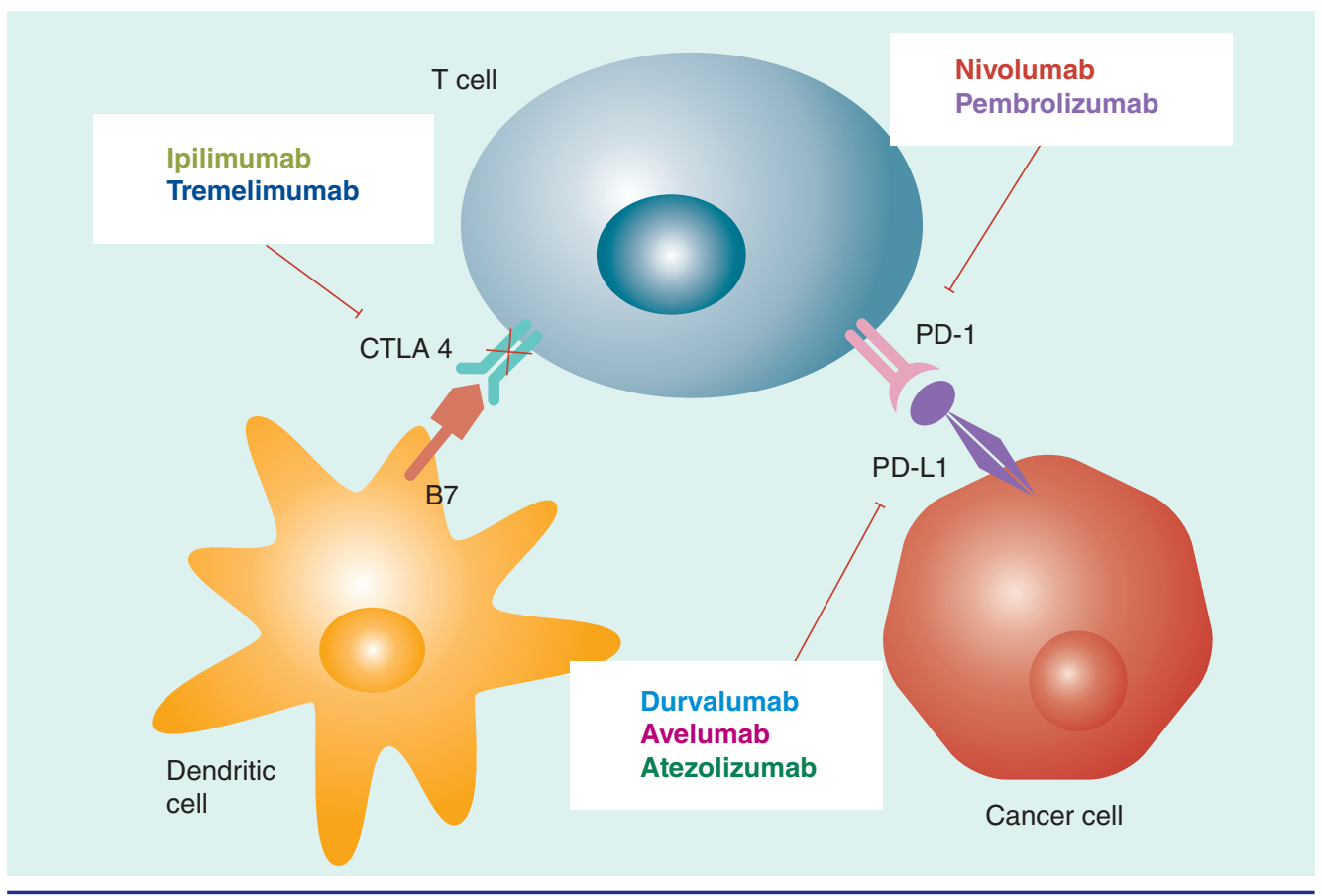

Figure 2. Mechanism of action of the immune checkpoint inhibitors currently under investigation as potential systemic treatments for hepacellular carcinoma Immune evasion of tumor cells can be obtained through the activation of the immune inhibitory molecule cytotoxic T-lymphocyteassociated protein 4 (CTLA-4) by intratumoral dendritic cells. Also, tumor cells may directly inhibit cytotoxic $t$ lymphocytes through overexpression of the programmed death ligand 1 (PD-L1) molecule. This molecule binds to the programmed death 1 (PD1) receptor on cytotoxic T-lymphocytes leading to their apoptosis. Ipilimumab and tremelimumab bind to and inactivate CTLA-4 preventing its activation. PD1-PD-L1 binding may be preventing by blocking either PD1 (nivolumab, pembrolizumab) or PD-L1 (durvalumab, avelumab, atezolizumab).

in advanced liver cancer. Across dose escalation and expansion phases (262 patients), grade $3 / 4$ treatment-related adverse events occurred in $20 \%$. The ORR was 20\% (95\% CI: 15-26) in 214 patients treated in the dose expansion phase with a median duration of response of 9.9 months and a disease control rate of $64 \%$ (95\% CI: 58-71) [35].

Results from subsequent comparative, randomized cohorts of CheckMate 040 will further inform the therapeutic potential of nivolumab in patients with advanced HCC. The enrollment in cohorts 3 and 4 is currently closed. The analysis of the main end points (especially safety and ORR) is ongoing [33] and the final results are awaited with great expectations. The enrollment is still open only in cohort 5, which includes Child-Pugh B7-8 patients (both sorafenib-naive and pretreated).

It is worth noting that nivolumab is not the only checkpoint inhibitor that obtained positive results as HCC systemic treatment. In the setting of an investigator-initiated Phase II open-label, multicenter clinical trial, Sangro and collaborators recruited 21 patients with $\mathrm{HCV}$ related HCC. Most patients (57.1\%) were in the advanced stage and had not received sorafenib (76.2\%). Notably, $43 \%$ of patients were in Child-Pugh B class [36].

Enrolled patients were treated with tremelimumab until tumor progression or unacceptable toxicity. Objective response and disease control rate were 76.4 and $17.6 \%$, respectively. Median TTP was 6.48 months (95\% CI: 3.95-9.14). No toxicities requiring systemic steroid treatment were registered [36].

Additionally. Phase II trials of pembrolizumab in the second-line setting are also ongoing (NCT02658019, NCT02702414), as is a combination trial with tremelimumab and durvalumab (NCT02519348). Thanks to the promising preliminary results of Phase II immunotherapy trials, currently two different Phase III trials are undergoing, evaluating nivolumab 
versus sorafenib in the frontline setting [37] and pembrolizumab versus best supportive care in a second-line scenario, respectively [38].

\section{Galunisertib (TGF- $\beta$ inhibitor)}

Galunisertib is a oral small molecule inhibitor of the transforming growth factor- $\beta$ receptor I kinase (TGF- $\beta$ RI). The role of TGF- $\beta$ signaling in cancer is particularly complex (Figure 1) and includes actions on different hallmarks of cancer, including tumor proliferation, angiogenesis, invasion and metastasis and escape of immune surveillance [39]. Furthermore, TGF- $\beta$ pathway has been recently found to be involved in chemotherapy resistance to 5-fluorouracil and to the EGFR-TKI gefitinib [40].

Galunisertib is being investigated in patients with cancer with high unmet medical needs, including HCC. A Phase II dose-comparison study recruited HCC patients who had progressed or were ineligible for sorafenib, requiring AFP $\geq 1.5 \times$ upper normal limit as a required criterion (NCT02240433). The most interesting finding of this study was a median OS of approximately 21 months for patients who achieved at least a $20 \%$ reduction from baseline in AFP (25\% of the initial cohort of 191 patients) [41].

A Phase $\mathrm{Ib} / 2$ clinical trial is currently enrolling HCC patients refractory to sorafenib to evaluate the safety of the combination galunisertib+nivolumab (NCT02423343). Finally, another open-label Phase II clinical study is enrolling both naive and previously sorafenib-treated patients testing the combination galunisertib plus ramucirumab (NCT01246986).

\section{Metronomic capecitabine}

Conventional chemotherapies administered according to standard protocols (e.g., doxorubicin, oxaliplatin) have systematically failed to extend life expectancy of advanced HCC patients. Consequently, they have been outshadowed by target therapies and currently play a very limited role in HCC [42].

Where standard protocols failed, metronomic regimes (i.e., the continuous administration of low doses without prolonged drug-free breaks) [43] may obtain better results.

The potential efficacy of metronomic chemotherapy depends on several mechanisms: inhibition of tumoral angiogenesis; reduction of the therapeutic resistance of the tumor; and activation of the adaptive and innate immune response $[44,45]$.
In the field of HCC, metronomic capecitabine (MC) was evaluated in three different studies, mainly in a second-line setting [46-48]. All of these studies showed a low rate of adverse events and a good efficacy [46-48].

Still, the lack of prospective RCTs provide relevant limitations in the assessment of the efficacy of MC. These studies would require a large amount of independent funds, as capecitabine patent has expired.

Answering these calls from literature, the Italian Medicines Agency recently financed a cost-effectiveness, randomized, cross-over study comparing capecitabine versus sorafenib. The primary end point of this primary study will be evaluated in a first-line setting. However, the trial will also provide information about the effectiveness of $\mathrm{MC}$ as second-line therapy after sorafenib in the setting of a randomized trial [49].

\section{- Current challenges in the design of clinical trials}

The successful RESORCE trial is going to deeply affect the development of future clinical trials directed to patients who failed sorafenib.

Lessons learned both from the RESORCE and from previously failed RCTs will be helpful in the design of future studies, as minimal errors in the design of a trial can lead to its ultimate failure. In the following paragraphs we summarize the most controversial points affecting the design of postsorafenib trials.

\section{Stratification of the patients}

Determinants of postsorafenib survival

The main challenge for a postsorafenib trial is a correct identification of the biological factors involved in the post-treatment survival. This is a particularly critical process since HCC has a strongly heterogeneous natural history and biology [50]. Information about this topic are found in two brilliant papers by Reig et al. [51] and Iavarone et al. [52]. Reig et al. [51] prospectively followed 147 HCC patients treated with sorafenib, assessing their radiological progression at week 4 and then every 8 weeks using the Response Evaluation Criteria In Solid Tumors (RECIST) version 1.1. Four different patterns of progression were described: intrahepatic increase in tumor size, extrahepatic increase in tumor size, new intrahepatic lesions, new extrahepatic lesions. Factors independently associated with a worse OS included baseline BCLC C, compromised performance status, liver function 
deterioration, permanent sorafenib withdrawal, TTP and development of new extrahepatic lesion(s). Therefore, the authors identified three groups with different prognosis at the time of progression: BCLC B, BCLC C without new extrahepatic lesions and BCLC C with new extrahepatic lesions. The authors suggested that it is necessary to refine the BCLC definitions at the time of radiologic progression to properly predict the prognosis of patients still fit to enter into second-line studies.

Iavarone and colleagues [52] analyzed the predictors of OS in patients who permanently discontinued sorafenib. Analyzing 200 patients potentially eligible for second-line therapy, postsorafenib survival was dependent on reasons of discontinuation (intolerance, progressive disease, liver function deterioration), performance status, macrovascular invasion and extrahepatic spread. In particular, discontinuation due to adverse effects was associated with the best prognosis. This is a critical point in the design of the trials. The RESORCE trial was the first trial including only patients progressing to sorafenib [15], as such the population was more homogeneous and the confounding factor represented by the reason for sorafenib withdrawal was avoided. For trials enrolling both intolerant and progressed patient, the most significant inference deriving from Iavarone findings is the need for a stratification according to the reason of sorafenib withdrawal.

A final note of interest is represented by patients discontinuing sorafenib due to liver function deterioration. These patients have a grim prognosis and, at the same time, are not usually accepted in second-line trials to minimize the risk of noncancer-related death due to progressive hepatic failure [52]. In general, the opportunity of systemic treatments (including sorafenib) in Child-Pugh B patients is still a controversial issue $[17,18,53]$. These aspects leave Child-Pugh B patients as an orphan category (at the present time only a dedicated cohort of the nivolumab trial is enrolling these patients in a frontline and second-line setting [33]). A careful assessment of the parameters that entail the Child-Pugh B status should be considered in future studies to identify selected patients who can potentially benefit from study drugs.

\section{Number of previous lines of therapy}

Some of the so called 'second-line trials' actually offer the chance to enroll patients previously treated with two different systemic drugs [27] or without any limit of previous treatments [33]. On one hand, multiple previous therapeutic lines may not be seen as a significant problem due to the naturally reduced life expectancy (mainly related to compromised liver function over time). On the other hand, patients who underwent multiple lines are usually more frail, even if they respect the inclusion criteria in terms of liver functioning and performance status. Two different solutions can be adopted to avert possible biases. First, future trials may allow only one (sorafenib) or two previous lines (sorafenib and regorafenib) of therapy, imitating most of the current trials. Second, enrollment could be granted without limit of previous therapies (thus granting more eligible patients) but stratifying patients according to the number of previous therapeutic lines.

\section{Macrovascular invasion/extrahepatic spread:}

the 'and/or' dilemma

The SHARP trial stratified patients according to geographic region, performance status and presence/absence of macrovascular invasion or extra hepatic spread [2]. Following its success, most of the subsequent trials have been designed on the same model. Eventually, the combined stratification for macrovascular invasion or extrahepatic led to severe issues in the BRISK trial [8]. Analyzing the possible causes of its failure, it was noted that a much larger number of patients with macrovascular invasion had been randomized into brivanib treatment arm. This imbalance was masked by the fact that the majority of patients had extrahepatic spread and thus favored the control arm [8,54].

Furthermore, the aforementioned study by Iavarone clearly demonstrated that both macrovascular invasion and extrahepatic spread are predictive of OS [52]. Lessons from this bias were learned by the authors of some of the subsequent trials, which considered the two factors separately. The successful RESORCE trial is one of these cases [15]. However, some trials still adopt the same design of the SHARP, jeopardizing a possibly positive outcome of the study.

\section{The quest for biomarkers}

The identification of biomarkers predictive of favorable response is the Holy Grail in Oncology trials. Obviously, the availability of biomarkers of response allow a tailored approach to the single patients, avoiding unnecessary and potentially harmful treatments to patients who are 
unlikely to respond. Further, analyzing HCC tumor specimens is also of considerable help to improve the knowledge about development and progression of HCC [55]. To date, no reliable biomarkers has been found for sorafenib [56]. Interestingly, in the original REACH trial [9], AFP levels of at least $400 \mathrm{ng} / \mathrm{ml}$ were associated with a significant survival benefit deriving from ramucirumab. Similarly, in the Phase II tivantinib trial, a survival benefit from tivantinib was observed only in the MET-positive patients [57]. Even more interestingly, METpositivity rate was $40 \%$ in tumoral samples obtained before sorafenib treatment and over $80 \%$ in samples obtained after progression to sorafenib. Even if the benefits of tivantinib were not confirmed in two subsequent Phase III trials $[13,14]$, the biomarker evaluation provided interesting information. For instance, MET activation was confirmed as one of the alternative escape routes found by tumoral cells during exposition to antiangiogenic drugs [58]. As for immunotherapy, tumor PD-L1 expression was regarded as a possible biomarker but preliminary experiences with nivolumab seem not to confirm this hypothesis [35].

Biomarkers can be identified either in peripheral blood or in tumor tissue. The access to peripheral blood samples is easy and riskless. On the contrary, obtaining samples from liver biopsies is more problematic and comes with risks of procedural complications. The opportunity of liver biopsies is a hot topic in hepatic oncology and has been recently object of a debate between eminent experts in the field $[59,60]$. The clinical and biological heterogeneity of patients with advanced HCC and the need for biomarkers will probably lead to a 'back to the future' scenario, with a predictable trend toward an increase in the number of liver biopsies in patients eligible for clinical trials [60].

Recently, a multisocietary position paper by the Italian Association for the Study of the Liver and the Italian Association of Medical Oncology endorsed this approach and described as ethically acceptable the procedure of collecting new tumor samples after progression to sorafenib, in the setting of clinical trials [61].

Unfortunately, not all the tumors are located in safely reachable locations. Also, some patients may be unwilling to undergo an invasive procedure and prefer to be enrolled in a different trial or treated with off-label drugs. In this scenario, the designers of RCTs will face a choice between a larger and easier recruitment and the chance to find novel biomarkers of response.

\section{Radiologic criteria of response}

In oncology, OS is the most important end point. However, surrogate end points are needed both in clinical and in research practices. Radiologic criteria of response are the most frequently used surrogate end points, sometimes also representing the principal end point of Phase II trials. However, there is not a universal consensus on which criteria best predict the clinical outcome.

When the SHARP study was designed, RECIST were the only universally accepted criteria [62]. After a few years, it became clear that sorafenib can induce a temporary increase in target lesions, with the theoretical possibility of a pseudoprogression in a way similar to transarterial chemoembolization [63]. Further, some aspects regarding the assessment of new intrahepatic lesions, lymphnodes and ascites were ill-suited for the evaluation of cirrhotic patients. Modified RECIST criteria for liver disease (mRECIST) were thus proposed in order to better assess intratumoral changes following therapeutic procedures, both locoregional and systemic [64].

In the latter setting, mRECIST are able to identify a proportion of patients with a better prognosis in which sorafenib obtain a 'vascular shutdown' of the nodules and whose response would have been otherwise classified as 'stable disease' according to RECIST [65]. However, mRECIST and RECIST are equivalent in the evaluation of progression, which is the most important end point as regards to therapeutic decisions [65].

A very recent and interesting analysis on the population of the second-line Phase III BRISK study showed that response according to the mRECIST was an independent predictor of OS in patients treated with anti-VEGFR agents [66]. However, the performance of mRECIST was not compared with RECIST. Thus, it remains to be established that mRECIST is superior to RECIST in the setting of systemic therapy, as the only study showing their benefit is a retrospective analysis in 53 patients $[65,67]$.

Currently, regulatory agencies still recognize RECIST as the reference for the evaluation of response and more evidences are needed before mRECIST can be considered as the preferred criteria for drug development in advanced HCC [67]. 
New challenges in this field will come from the immunotherapy trials. Seminal ipilimumab trials in melanoma showed that up to $40 \%$ of patients who achieved a significant OS benefit had received an initial response of progressive disease according to RECIST 1.1 [68-70].

This pseudoprogression was due to the delayed effect of immunotherapeutic drugs (whose action may require weeks to be appreciated) and to the lymphocytic infiltration and inflammation of tumor nodules [71,72].

Thus, a panel of expert proposed the immunerelated response criteria (irRC) in 2009 [73]. According to irRC, new lesions may still develop in the first 12 weeks of treatment without automatically defining progressive disease. Further, new lesions should be confirmed 4 weeks after the first documentation of apparent progression [73]. A slight revision of the irRC has been recently announced; however, these recommendations were confirmed [74].

\section{Treatment beyond radiologic progression}

The aforementioned uncertainties surrounding radiologic criteria generate a further doubt: should investigational drugs be withdrawn at the first occurrence of radiologic progression?

In 2008 the SHARP trial was stopped after the second interim analyses due to a statistically significant difference in OS in the two arms [2]. The authors made clear that the statistical significance was obtained also thanks to the possibility to treat patients even beyond radiologic progression, provided that the Investigator believed it was beneficial for the patient. In fact, at the interim analyses, the statistical significance was not met in the subgroup of patients who were discontinued after radiologic evidence of progressive disease [2]. The benefit of treatment beyond progression was subsequently confirmed by clinical evidences [75]. Further, a recent combined analysis of the two registrative sorafenib trials showed no correlation between TTP and OS [76]. However, the RESORCE trial regarded to radiologic progression as defined by $\mathrm{mRE}$ CIST as a stopping rule [9]. The discordance between TTP and OS is now a very controversial topic in other types of cancer [77] and will probably remain an open problem in the design of future trials.

\section{End points of the studies}

There is a substantial agreement about the opportunity of setting OS as the principal end-point in Phase III trials. Instead, the situation is more problematic in Phase II studies. In this setting, intermediate end points such as ORR and TTP are usually preferred [78], as waiting for OS assessment is time-consuming and may delay the development of an effective therapy. There are, however, reasons against this strategy. Experiences from previous immunotherapy trials, such as CTLA-4 inhibitors for treatment of metastatic melanoma and non-small-cell lung carcinoma, teach us that in these cases TTP and ORR underestimated the benefit deriving from these drugs [43,71-72]. Due to a possible delayed response to therapy (with a consequent condition of pseudoprogression), some patients performed poorly in terms of TTP and ORR but achieved a significant benefit in terms of OS nonetheless [71,72]. Therefore, intermediate end points are not validated parameters in the setting of immunotherapy and should be used with caution as principal end points, even in Phase II studies. In particular, the combined choice of ORR as primary end point and of RECIST 1.1 as radiologic criteria of response may represent a very critical issue, potentially leading to an unmet primary end point.

\section{Control group}

As the RESORCE trial was announced to have reached the OS end-point [15], future randomized second-line trials against placebo may not be ethical. Patients progressing to sorafenib should receive regorafenib or be included in head-tohead trials comparing investigational study drugs versus regorafenib. Still, there are more uncertainties about patients intolerant to sorafenib, which may become an orphan group. More complexities will arise in case of success of one or more of the current Phase III trials, potentially leading to complicated algorithms to identify the better control group in future studies.

\section{Conclusion}

HCC is a malignancy characterized by a great biological heterogeneity. As such, trials with only few stratification factors are at risk of failure, as seen in the past. The RESORCE trial is an example of a comprehensive evaluation of these factors. Further, it analyzed a more homogenous study population enrolling only patients progressed to sorafenib. These factors strongly contributed to its brilliant success.

Obviously, more stratification factors mean a greater number of patients to be enrolled 
and greater costs: however, eventual failure of the trial would entail even greater economic consequences.

A great variety of drugs are currently being investigated in patients who were unresponsive or intolerant to sorafenib. Among ongoing clinical trials, immunotherapy trials are increasingly gaining the attention of hepatic oncologists. As such, the design of dedicated Phase II clinical trials will need particular attention in the choice of the most appropriate radiological response criteria and primary end points.

Lessons learned from previously failed trials and from the successful RESORCE trial will probably help in the creation of more adequate study designs and to the development of a larger therapeutic arsenal for advanced HCC patients.

\section{Future perspective}

Most of currently ongoing postsorafenib trials involve immunotherapeutic drugs. The success or the failure of this class of drugs is destined to shape the future of the HCC systemic treatment in the next 5-10 years. In case of success, a new era of head-to-head trials with the now dominating antiangiogenic drugs will probably characterize the next years. Should these drugs fail, the future will be similar to the present days, predictably with more and more trials exploring drugs targeted to specific neoplastic pathways, with or without a combined anti-VEGFR blockade. Notably, some of the most critical point of study design may particularly afflict immunotherapy trials. Consequently, a refinement of study protocols will also gain an increased importance in the near future, as seemingly little errors can dictate an unfavorable outcome.

\section{Author contributions}

F Tovoli, S De Lorenzo and G Brandi designed the study and analyzed the data; A Palloni, G Frega and I Garajova performed the search about previous and ongoing trials; MA Barbera, MA Pantaleo and G Biasco critically analyzed the findings in the light of recent literature in the field. All of the authors contributed to correction and editing of the final manuscript. All authors approved the final draft prior to submission.

Financial \& competing interests disclosure The authors have no relevant affiliations or financial involvement with any organization or entity with a financial interest in or financial conflict with the subject matter or materials discussed in the manuscript. This includes employment, consultancies, honoraria, stock ownership or options, expert testimony, grants or patents received or pending, or royalties.

No writing assistance was utilized in the production of this manuscript.

\section{EXECUTIVE SUMMARY}

\section{Currently ongoing trials}

- Three postsorafenib Phase III randomized clinical trials are currently ongoing (ramucirumab, cabozantinib, pembrolizumab).

- Although not in a pure postsorafenib setting, other molecules are under investigations in Phase II or III trials (galunisertib, capecitabine).

- Immunotherapy trials now represent a consistent part of postsorafenib trials.

\section{Current challenges in the design of clinical trials}

- Flaws in the design partially influenced some previous randomized clinical trial failures and still persist in ongoing trials.

- An accurate stratification of the patients is difficult but of utmost importance and should rely on many factors.

- Biomarkers will probably play a pivotal role in the future, leading to an increased number of liver biopsies.

- Uncertainties still surround the radiologic criteria of response, especially for immunotherapy. As such, end points such as overall response rate and time to progression should be carefully used.

\section{Conclusion}

- Hepatocellular carcinoma is characterized by a great biological heterogeneity. As such, trials with only few stratification factors or other flaws are at risk of failure, as seen in the past.

- The foreseeable future will be deeply influenced by the results of immunotherapy trials, whose design will need particular attention in well-defined critical points. 
Open access

This work is licensed under the AttributionNonCommercial-NoDerivatives 4.0 Unported License. To view a copy of this license, visit http://creativecommons.org/

licenses/by-nc-nd/4.0/

\section{References}

Papers of special note have been highlighted as:

- of interest; $\bullet$ of considerable interest

1 Ferlay J, Soerjomataram I, Ervik M et al. GLOBOCAN $2012 \mathrm{v} 1.0$, cancer incidence and mortality worldwide: IARC CancerBase No. 11 Lyon, France: International Agency for Research on Cancer. http://globocan.iarc.fr

2 Llovet JM, Ricci S, Mazzaferro V et al. Sorafenib in advanced hepatocellular carcinoma. N. Engl. J. Med. 359(4), 378-390 (2008).

3 European Association For The Study Of The Liver; European Organisation For Research And Treatment Of Cancer. EASL-EORTC clinical practice guidelines: management of hepatocellular carcinoma. J. Hepatol. 56(4), 908-943 (2012).

4 Kudo M, Izumi N, Kokudo N et al. Management of hepatocellular carcinoma in Japan: consensus-based clinical practice guidelines proposed by the Japan Society of Hepatology (JSH) 2010 updated version. Dig. Dis. 29(3), 339-364 (2011)

5 NCCN Clinical Practice Guidelines in Oncology. Hepatobiliary Cancer. V1 (2015). www.nccn.org

6 Omata M, Lesmana LA, Tateishi R et al. Asian Pacific Association for the Study of the Liver consensus recommendations on hepatocellular carcinoma. Hepatol Int. 4(2), 439-474 (2010).

7 Iavarone M, Cabibbo G, Piscaglia F et al. Field-practice study of sorafenib therapy for hepatocellular carcinoma: a prospective multicenter study in Italy. Hepatology 54(6), 2055-2063 (2011).

8 Llovet JM, Decaens T, Raoul JL et al. Brivanib in patients with advanced hepatocellular carcinoma who were intolerant to sorafenib or for whom sorafenib failed: results from the randomized Phase III BRISK-PS study. J. Clin. Oncol. 31(28), 3509-3516 (2013).

9 Zhu AX, Park JO, Ryoo BY et al. Ramucirumab versus placebo as second-line treatment in patients with advanced hepatocellular carcinoma following first-line therapy with sorafenib (REACH): a randomised, double-blind, multicentre, Phase III trial. Lancet Oncol. 16(7), 859-870 (2015).
10 Zhu AX, Kudo M, Assenat E et al. Effect of everolimus on survival in advanced hepatocellular carcinoma after failure of sorafenib: the EVOLVE-1 randomized clinical trial. JAMA 312(1), 57-67 (2014).

11 Abou-Alfa GK, Puig O, Daniele B et al. Randomized Phase II placebo controlled study of codrituzumab in previously treated patients with advanced hepatocellular carcinoma. J. Hepatol. 65(2), 289-295 (2016).

12 Abou-Alfa GK, Qin S, Ryoo BY et al. Phase III randomized study of second line ADI-peg 20 (A) plus best supportive care versus placebo $(\mathrm{P})$ plus best supportive care in patients (pts) with advanced hepatocellular carcinoma (HCC). J. Clin. Oncol. 34(Suppl.), Abstract 4017 (2016)

13 Daiichi Sankyo Press Release. www.daiichisankyo.com

14 ArQule Press Release. http://investors.arqule.com

15 Bruix J, Qin S, Merle P et al. Regorafenib for patients with hepatocellular carcinoma who progressed on sorafenib treatment (RESORCE): a randomised, double-blind, placebo-controlled, Phase III trial. Lancet 389(10064), 56-66 (2016).

-• Illustrates the results of the first successful second-line trial for hepatocellular carcinoma (HCC).

16 Eisai Press Release. http://eisai.mediaroom.com

17 Marrero JA, Kudo M, Venook AP et al. Observational registry of sorafenib use in clinical practice across Child-Pugh subgroups: the GIDEON study. J. Hepatol. 65(6), 1140-1147 (2016).

18 Granito A, Bolondi L. Non-transplant therapies for patients with hepatocellular carcinoma and Child-Pugh-Turcotte class B cirrhosis. Lancet Oncol. 18(2), e101-e112 (2017).

19 Forner A, Reig M. Does ramucirumab deserve a second chance for liver cancer? Lancet Oncol. 16(7), 751-752 (2015).

20 Sennino B, Ishiguro-Oonuma T, Wei Y et al. Suppression of tumor invasion and metastasis by concurrent inhibition of c-Met and VEGF signaling in pancreatic neuroendocrine tumors. Cancer Discov. 2(3), 270-287 (2012).
21 Ciamporcero E, Miles KM, Adelaiye R et al. Combination strategy targeting VEGF and $\mathrm{HGF} / \mathrm{c}-\mathrm{met}$ in human renal cell carcinoma models. Mol. Cancer Ther. 14(1), 101-110 (2015).

22 You H, Ding W, Dang H et al. c-Met represents a potential therapeutic target for personalized treatment in hepatocellular carcinoma. Hepatology 54(3), 879-889 (2011).

23 Daudigeos-Dubus E, Le Dret L, Bawa O et al. Dual inhibition using cabozantinib overcomes HGF/MET signaling mediated resistance to pan-VEGFR inhibition in orthotopic and metastatic neuroblastoma tumors. Int. J. Oncol. 50 (1), 203-211 (2017).

24 Yakes FM, Chen J, Tan J et al. Cabozantinib (XL184), a novel MET and VEGFR2 inhibitor, simultaneously suppresses metastasis, angiogenesis, and tumor growth. Mol. Cancer Ther. 10(12), 2298-2308 (2011).

25 Elisei R, Schlumberger MJ, Muller SP et al. Cabozantinib in progressive medullary thyroid cancer. J. Clin. Oncol. 31(29), 3639-3646 (2013).

26 Cohn AL, Kelley RK, Yang TS et al. Activity of cabozantinib (XL184) in hepatocellular carcinoma patients (pts): results from a Phase II randomized discontinuation trial (RDT).J. Clin.Oncol. 30(4_Suppl.), 261 (2012).

27 Abou-Alfa GK, Cheng AI, Meyer T et al. Phase III randomized, double-blind, controlled study of cabozantinib (XL184) versus placebo in subjects with hepatocellular carcinoma who have received prior sorafenib (CELESTIAL; NCT01908426). J. Clin. Oncol. 32 (5 Suppl.), Abstract TPS4150 (2014).

28 Lesokhin AM, Callahan MK, Postow MA et al. On being less tolerant: enhanced cancer immunosurveillance enabled by targeting checkpoints and agonists of $\mathrm{T}$ cell activation. Sci. Transl. Med. 7(280), 280sr1 (2015).

29 Mittal D, Gubin MM, Schreiber RD et al. New insights into cancer immunoediting and its three component phases-elimination, equilibrium and escape. Curr. Opin. Immunol. 27, 16-25 (2014).

30 Larkin J, Chiarion-Sileni V, Gonzalez R et al. Combined nivolumab and Ipilimumab or monotherapy in untreated melanoma. N. Engl. J. Med. 373(1), 23-34 (2015) 
- Seminal paper in the immunotherapy field.

31 Borghaei H, Paz-Ares L, Horn L et al. Nivolumab versus docetaxel in advanced nonsquamous non-small-cell lung cancer. N. Engl. J. Med. 373(17), 1627-1639 (2015).

32 Brahmer J, Reckamp KL, Baas P et al. Nivolumab versus docetaxel in advanced squamous-cell non-small-cell lung cancer. N. Engl. J. Med. 373(2), 123-135 (2015).

33 Clinical Trials Database: NCT01658878. https://clinicaltrials.gov

34 Kudo M. Immune checkpoint inhibition in hepatocellular carcinoma: basics and ongoing clinical trials. Oncology 92(Suppl. 1), 50-62 (2017).

- Detailed review about ongoing immunotherapy trials in HCC.

35 El-Khoueiry AB, Sangro B, Yau T et al. Nivolumab in patients with advanced hepatocellular carcinoma (CheckMate 040): an open-label, non-comparative, Phase I/II dose escalation and expansion trial. Lancet doi:10.1016/S0140-6736(17)31046 (2017) (Epub ahead of print).

-. Large Phase Ib/II trial of immunotherapy in HCC.

36 Sangro B, Gomez-Martin C, de la Mata M et al. A clinical trial of CTLA-4 blockade with tremelimumab in patients with hepatocellular carcinoma and chronic hepatitis C. J. Hepatol. 59(1), 81-88 (2013).

37 Sangro B, Park JW, Dela Cruz CM et al. A randomized, multicenter, Phase III study of nivolumab vs sorafenib as first-line treatment in patients (pts) with advanced hepatocellular carcinoma (HCC): CheckMate. J. Clin. Oncol. 34(Suppl.), Abstract TPS4147 (2016).

38 Finn RS, Chan SL, Zhu AX et al. KEYNOTE-240: Randomized Phase III study of pembrolizumab versus best supportive care for second-line advanced hepatocellular carcinoma. J. Clin. Oncol. 35(Suppl. 4S), Abstract TPS503 (2017).

39 Herbertz S, Sawyer JS, Stauber AJ et al. Clinical development of galunisertib (LY2157299 monohydrate), a small molecule inhibitor of transforming growth factor-beta signaling pathway. Drug Des. Dev. Ther. 9, 4479-4499 (2015).

40 Loeffler M, Kruger JA, Niethammer AG et al. Targeting tumor-associated fibroblasts improves cancer chemotherapy by increasing intratumoral drug uptake. J. Clin. Invest. 116(7), 1955-1962 (2006).

41 Faivre S, Santoro A, Kelley RK et al. A Phase II study of a novel transforming growth factor-beta (TGF- $\beta$ ) receptor I kinase inhibitor, LY2157299 monohydrate, in patients with advanced hepatocellular carcinoma (HCC). Presented at: Gastrointestinal Cancers Symposium. San Francisco, CA, USA 16-18 January 2014.

42 Stotz M, Gerger A, Haybaeck J et al. Molecular targeted therapies in hepatocellular carcinoma: past, present and future. Anticancer Res. 35(11), 5737-5744 (2015).

43 Von Delius S, Lersch C, Mayr M et al. Capecitabine for treatment of advanced hepatocellular carcinoma.

Hepatogastroenterology 54(80), 2310-2314 (2007).

44 Pasquier E, Kavallaris M, André N. Metronomic chemotherapy: new rationale for new directions. Nat. Rev. Clin. Oncol. 7(8), 455-465 (2010).

45 Kareva I, Waxman DJ, Lakka Klement G. Metronomic chemotherapy: an attractive alternative to maximum tolerated dose therapy that can activate anti-tumor immunity and minimize therapeutic resistance. Cancer Lett. 358(2), 100-106 (2015).

46 Brandi G, de Rosa F, Agostini V et al. Metronomic capecitabine in advanced hepatocellular carcinoma patients: a Phase II study. Oncologist 18(12), 1256-1257 (2013).

47 Granito A, Marinelli S, Terzi E et al. Metronomic capecitabine as second-line treatment in hepatocellular carcinoma after sorafenib failure. Dig. Liver Dis. 47(6), 518-522 (2015).

48 Casadei GA, Foca F, Scartozzi M et al. Metronomic capecitabine versus best supportive care as second-line treatment in hepatocellular carcinoma: a retrospective study. Sci. Rep. 7, 42499 (2017).

49 A multicenter, randomized, open-label Phase III study of two anti-angiogenic strategies in advanced hepatocellular carcinoma patients with cross-over at first-line failure: metronomic Capecitabine/Sorafenib (Arm A) vs Sorafenib/metronomic Capecitabine (Arm B). www.agenziafarmaco.gov.it

50 Cabibbo G, Enea M, Attanasio M et al. A meta-analysis of survival rates of untreated patients in randomized clinical trials of hepatocellular carcinoma. Hepatology 51(4), 1274-1283 (2010).

51 Reig M, Rimola J, Torres F et al. Postprogression survival of patients with advanced hepatocellular carcinoma: rationale for second-line trial design. Hepatology 58(6), 2023-2031 (2013).
- Seminal paper for the design of future postsorafenib trials.

52 Iavarone $\mathrm{M}$, Cabibbo $\mathrm{G}$, Biolato $\mathrm{M}$ et al. Predictors of survival in patients with advanced hepatocellular carcinoma who permanently discontinued sorafenib. Hepatology 62(3), 784-791 (2015).

- Seminal paper for the design of future postsorafenib trials.

53 Hollebecque A, Cattan S, Romano O et al. Safety and efficacy of sorafenib in hepatocellular carcinoma: the impact of the Child-Pugh score. Aliment. Pharmacol. Ther. 34(10), 1193-1201 (2011).

54 Bolos D, Finn RS. Systemic therapy in HCC: lessons from brivanib. J. Hepatol. 61(4), 947-950 (2014).

55 Rimassa L, Reig M, Abbadessa G et al. Tumor biopsy and patient enrollment in clinical trials for advanced hepatocellular carcinoma. World J. Gastroenterol. 23(13), 2448-2452 (2017).

56 Shao YY, Hsu CH, Cheng AL. Predictive biomarkers of sorafenib efficacy in advanced hepatocellular carcinoma: are we getting there? World J. Gastroenterol. 21(36), 10336-10347 (2015).

57 Santoro A, Rimassa L, Borbath I et al. Tivantinib for second-line treatment of advanced hepatocellular carcinoma: a randomized, placebo controlled Phase 2 study. Lancet Oncol. 14(1), 55-63 (2013).

58 Rimassa L, Abbadessa G, Personeni N et al. Tumor and circulating biomarkers in patients with second-line hepatocellular carcinoma from the randomized Phase II study with tivantinib. Oncotargets 7(45), 72622-72633 (2016).

- Interesting paper about biomarkers in HCC.

59 Sherman M, Bruix J. Biopsy for liver cancer: how to balance research needs with evidence-based clinical practice. Hepatology 61(2), 433-436 (2015).

60 Torbenson M, Schirmacher P. Liver cancer biopsy - back to the future?! Hepatology 61(2), 431-433 (2015).

61 Italian Association for the Study of the Liver. Multisocietary recommendations for the management of patients with hepatocellular carcinoma. www.webaisf.org

62 Therasse P, Arbuck SG, Eisenhauer EA et al. New guidelines to evaluate the response to treatment in solid tumors. European Organization for Research and Treatment of Cancer, National Cancer Institute of the United States, National Cancer Institute of Canada. J. Natl Cancer Inst. 92(3), 205-216 (2000). 
63 Bruix J, Sherman M, Llovet JM et al. Clinical management of hepatocellular carcinoma. Conclusions of the Barcelona-2000 EASL conference. J. Hepatol. 35(3), 421-430 (2001).

64 Lencioni R, Llovet JM. Modified RECIST (mRECIST) assessment for hepatocellular carcinoma. Semin. Liver Dis. 30(1), 52-60 (2010).

65 Edeline J, Boucher E, Rolland Y et al. Comparison of tumor response by Response Evaluation Criteria in Solid Tumors (RECIST) and modified RECIST in patients treated with sorafenib for hepatocellular carcinoma. Cancer 118(1), 147-156 (2012).

66 Lencioni R, Montal R, Torres F et al. Objective response by mRECIST as a predictor and potential surrogate end-point of overall survival in advanced HCC. J. Hepatol. 66(6), 1166-1172 (2017).

67 Edeline J, Palmer D, Blanc JF et al. mRECIST for systemic therapies: more evidence is required before recommendations could be made. J. Hepatol. doi:10.1016/j. jhep.2017.01 (2017) (Epub ahead of print).

O’Day SJ, Maio M, Chiarion-Sileni V et al. Efficacy and safety of ipilimumab monotherapy in patients with pretreated advanced melanoma: a multicenter single-arm Phase II study. Ann. Oncol. 21(8), 1712-1717 (2010).

69 Wolchok JD, Neyns B, Linette G et al. Ipilimumab monotherapy in patients with pretreated advanced melanoma: a randomised, double-blind, multicentre, Phase II, dose-ranging study. Lancet Oncol. 11(2), 155-164 (2010).

70 Weber J, Thompson JA, Hamid O et al. A randomized, double-blind, placebocontrolled, Phase II study comparing the tolerability and efficacy of ipilimumab administered with or without prophylactic budesonide in patients with unresectable stage III or IV melanoma. Clin. Cancer Res. 15(17), 5591-5598 (2009).

71 Hodi FS, Butler M, Oble DA et al. Immunologic and clinical effects of antibody blockade of cytotoxic T lymphocyteassociated antigen 4 in previously vaccinated cancer patients. Proc. Natl Acad. Sci. USA 105(8), 3005-3010 (2008).

72 Hodi FS, Oble DA, Drappatz J et al. CTLA-4 blockade with ipilimumab induces significant clinical benefit in a female with melanoma metastases to the CNS. Nat. Clin. Pract. Oncol. 5(9), 557-561 (2008).
73 Wolchok JD, Hoos A, O'Day S et al. Guidelines for the evaluation of immune therapy activity in solid tumors: immunerelated response criteria. Clin. Cancer Res. 15(23), 7412-7420 (2009).

74 Dolgin E. Cancer immunology community seeks better end points. Nat. Rev. Drug Discov. 15(12), 807-809 (2016).

75 Miyahara K, Nouso K, Morimoto Y. Efficacy of sorafenib beyond first progression in patients with metastatic hepatocellular carcinoma. Hepatol. Res. 44(3), 296-301 (2014).

76 Huang L, Yoriko DS, Minghua S et al. Weak correlation of overall survival and time to progression in advanced hepatocellular carcinoma. J. Clin. Oncol. 35(Suppl. 4S), Abstract 23 (2017).

77 Oxnard GR, Morris MJ, Hodi FS et al. When progressive disease does not mean treatment failure: reconsidering the criteria for progression. J. Natl Cancer Inst. 104(20), 1534-1541 (2012).

78 Llovet JM, Di Bisceglie AM, Bruix J et al. Design and end points of clinical trials in hepatocellular carcinoma. J. Natl Cancer Inst. 100(10), 698-711 (2008). 